\title{
Análisis económico de la violencia doméstica en Colombia, 2012-2015 ${ }^{1}$
}

\section{Economic analysis of domestic violence in Colombia, 2012-2015}

Recibido: 18 de julio de 2016 - Revisado: 22 de noviembre de 2016 - Aceptado: 15 de febrero de 2017.

Luis Eduardo Sandoval ${ }^{2}$

Maria Camila Otálora ${ }^{3}$

\section{Resumen}

Este documento parte de un modelo no cooperativo, en el cual si una mujer trabaja y el hogar presenta otros ingresos, se disminuyen las agresiones físicas hacia la mujer. Se usan variables que inciden en la violencia por parte del jefe del hogar y provocan daños en el vínculo cooperativo del padre hacia la mujer y la familia. Los resultados muestran que si la mujer tiene mayor educación, esta casada, lleva mas tiempo con su pareja, tiene mayor edad y el estrato social es más alto, se reduce la violencia física hacia la mujer. En casos de mujeres víctimas de agresiones físicas por parte del jefe del hogar, en su mayoría se trata de mujeres que están casadas y trabajan.

Palabras claves

Violencia doméstica, violencia de género, negociación en los hogares.

\begin{abstract}
This document is based on a non-cooperative model, in which if a woman works and the household presents other income, physical aggressions against women are reduced. Variables are used that influence violence by the head of the household and cause damage to the cooperative bond between the father and the woman and the family. The results show that if women have more education, are married, have more time with their partner, are older and their social stratum is higher, physical violence to women is reduced. In cases of women victims of physical aggression by the head of the household, the majority are women who are married and work.
\end{abstract}

\section{Keywords}

Domestic violence, gender violence, home negotiation.

\footnotetext{
${ }^{1}$ Este documento corresponde al proyecto "Violencia y victimización de género en Colombia" financiado por el área de investigaciones de la Escuela Militar de Cadetes.

${ }^{2}$ Profesor asociado del Departamento de Economía de la Universidad Militar Nueva Granada. Bogotá, Colombia.

Correo electrónico: luis.sandoval@ unimilitar.edu.co.

${ }^{3}$ Antropóloga con énfasis en salud Pontificia Universidad Javeriana.

Correo electrónico: kmixox@gmail. com

Para citar este artículo use: Sandoval, L., \& Otálora, M. (2017) Análisis económico de la violencia domestica en Colombia, 2012 2015. Civilizar Ciencias Sociales y Humanas, 17(33), 149-162. doi $10.22518 / 16578953.905$
} 


\section{Introducción}

La violencia se ha convertido en una forma inadecuada de comunicación humana y protagonista de las relaciones sociales; da cuenta tanto de relaciones de poder existentes, como de las estructuras jerarquizadas y jerarquizantes de las sociedades. Este fenómeno, observado en las pequeñas estructuras sociales que son las familias, permite encontrar divisiones sociales que pueden ser trasladadas a las estructuras más grandes de las sociedades.

Cada vez son más los estudios sobre violencia intrafamiliar que abarcan las diversas tipologías de familia que existen en la actualidad, más allá de la familia nuclear o su definición estatal de "célula básica de la sociedad". La violencia intrafamiliar se ha convertido en un tema de salud pública debido a que está relacionado con casos de feminicidio e implica la supervisión y acción de organismos de control y otras entidades y organizaciones estatales.

En consecuencia, y a partir del modelo planteado por Farmer \& Tiefenthaler (1997) que plantea vínculos entre hombre y mujer que componen un hogar, con la violencia doméstica, se propone una solución de equilibrio parcial donde cada cual, independiente de sus preferencias y puntos de congruencia, maximizen su nivel de utilidad. Pero el entorno no cooperativo, provocado por la violencia física inflingida a la mujer, modifica su comportamiento altruista, ella deja de tolerar este tipo de agresiones, y encuentra en el aumento de sus ingresos, mejorando sus condiciones económicas, una variación de la utilidad marginal por menor agresión por parte del jefe del hogar. Deberá determinarse, con base en la estática comparativa de los resultados, si es posible predecir que un aumento en el ingreso económico de la mujer y del hogar, sumado a un más alto nivel de escolaridad y al tiempo que se lleva en pareja, aumenta la probabilidad de dejar la relación y de esta forma, disminuir las agresiones físicas hacia la mujer y otros integrantes de la familia.
Para alcanzar lo anterior, nos hemos nutrido de estudios locales e internacionales sobre violencia en los hogares. A partir de ellos, proponemos un modelo teórico en el que planteamos incentivos para que las víctimas de violencia intrafamiliar mejoren su condición social y terminen las relaciones donde existen agresiones que destrozan la unidad familiar. Sugerimos también los equilibrios cooperativos, que buscan unir a personas que desean mejorar su bienestar social.

Se ha aplicado empíricamente el modelo arriba mencionado y junto con las variables manejadas, los resultados permiten plantear que las mujeres víctimas, una vez cuentan con ingresos propios y en consecuencia mejoran sus condiciones sociales, generan una variación hacia la baja, de las agresiones físicas. Sumado a esto, hemos tenido en cuenta otras condiciones intrínsecas de los hogares y que son determinantes de la violencia de género. Finalmente, se presentamos las conclusiones de los resultados encontrados, proponiendo futuras discusiones al respecto.

\section{Antecedentes}

En Colombia, aunque se ha reducido la intensidad del conflicto armado y se ha incrementado la presencia del Estado, lo que ha permitido aumentar la seguridad en las regiones, estas zonas presentan condiciones particulares que dan origen a formas de violencia relacionadas con las consecuencias del conflicto armado. También, se presentan actos de violencia que se explican por mayor densidad poblacional y condiciones económicas regionales (Gottfredson \& Hirschi, 1990). A este propósito, es necesario identificar en escenarios de posconflicto, la posibilidad de que se presenten nuevas formas de violencia de género como consecuencia de una baja seguridad y por problemáticas regionales (Acero, Escobar, \& Castellanos, 2007), así como por el contexto de los municipios de acuerdo con los recursos naturales predominantes. 
La transición de conflicto a posconflicto puede afectar de forma diferenciada a la población vulnerable, según el género y el lugar que ocupa cada persona en el hogar. Este tipo de violencia no se relaciona con el efecto del conflicto armado en las regiones, más bien se asocia con una violencia que se manifiesta por agresiones físicas o psicológicas y que afecta a las personas de acuerdo con su situación laboral, su orientación sexual o su, su salud sexual y reproductiva. Adicionalmente, las condiciones demográficas muestran mayor dinámica de ciertos tramos poblacionales a nivel municipal, y esto acarrera que las regiones sean propensas a la ocurrencia de conflictos sociales por cuenta de la mayor densidad de dichos grupos poblacionales juveniles (Boucekkine, Croix, \& Licandro, 2002). Advirtamos entonces, que las formas de violencia presentes en las regiones no solo son actos del conflicto armado sino también hechos que se deben a las condiciones internas y específicas de cada región y de los hogares, las cuales se describen en las siguientes secciones.

\section{Determinantes de violencia en los hogares.}

La violencia intrafamiliar ha buscado ser cuantificada a través de diferentes organismos de control estatal como las comisarías de familia, la Policía Nacional o el Instituto Nacional de Medicina Legal. Sin embargo los resultados de los informes de estas instituciones no reflejan el fenómeno de la violencia intrafamiliar en toda su proporción, dado que están condicionados a que la víctima denuncie y esto suele suceder cuando presenta visibles marcas del maltrato en su cuerpo.

Se han identificado tres fases dentro de la dinámica de la violencia intrafamiliar: en la primera, se acumulan tensiones y se generan agresiones violentas pero sin dejar marcas físicas; en la segunda, sucede la agresión física; en la tercera, se manifiesta arrepentimiento y perdón. Esta última, muy común en el caso de la violencia conyugal (Quiñones, Arias, Delgado, \& Tejera, 2011) y la más frecuente o la raíz de la violencia intrafamiliar.

La violencia doméstica podría ser prevenida e identificada antes de la marca corporal si existieran más redes de apoyo y políticas de prevención más que de sanción. El entorno (criminalidad, pobreza y principales actividades económicas) de las personas puede causar que los individuos, a cualquier edad, pasen a ejecutar actos de violencia (Chen, Thrane, Whitbeck, Johnson, \& Hoyt, 2007).

En este sentido, (Savoie, Bédard, \& Collins, 2006) analiza tasas de crimen a la propiedad y crimen violento con respecto a las características de un lugar en específico, considerando variables como el bajo ingreso, porcentaje de zona unifamiliar, multifamiliar y comercial así como la densidad de bares y el estado civil de las personas que habitan en el vecindario tal y como lo presenta (Watts, 1931). Los resultados muestran que características del barrio están asociadas con altas tasas de crímenes violencia y contra la propiedad, pero la fuerza de esta asociación puede variar cuando se toman en cuenta múltiples características en conjunto.

Adicionalmente las tasas de crimen violento son más bajas en barrios donde hay una gran proporción de residentes mayores de 20 años y con el estatus de comprometidos o casados y donde hay gran proporción de personas de bajos ingresos y solteras. El uso de zonas comerciales explica significativamente la variación en el crimen a la propiedad (Prendergast, Huang, \& Hser, 2008), así como, la densidad de bares está relacionada a mayor crimen (Anderson \& Diaz, 1996).

En contraste, se ha identificado que las tasas de crimen violento son más bajas en barrios donde hay una gran proporción de residentes mayores de 20 años, casados o en pareja, así como en zonas donde se concentran 
en su mayoría, personas de bajos ingresos y solteras.

La violencia intrafamiliar presenta diversas manifestaciones (Pallitto \& O'Campo, 2005), siendo el maltrato físico la expresión más denunciada puesto que su evidencia es notoria y fácil de demostrar ante los diferentes organismos de control estatal; sin embargo, el maltrato verbal, psicológico y emocional, que también son manifestaciones de la violencia intrafamiliar, son menos denunciados y asistidos porque sus efectos se suelen considerar menos graves (Pineda \& Otero, 2004). En Colombia, Medicina Legal registra los casos de violencia intrafamiliar diferenciados así: violencia de pareja; violencia contra niños, niñas y adolescentes; violencia entre otros familiares y violencia contra el adulto mayor. Estas formas de violencia, se relacionan con aspectos socioeconómicos y carencia de afecto en el hogar (Caicedo, 2005; Lafaurie, 2015).

$\mathrm{Si}$ bien la población pobre no necesariamente es la más violenta, si es la que más denuncia las agresiones; aún así, no hay que desconocer que la pobreza o la incertidumbre económica son un agravante en la conducta de los victimarios y las víctimas (Sánchez, Díaz, \& Formisano, 2003). Es necesario entender el orden de las diferentes estructuras familiares, ya que la mayoría de abusos violentos son ejecutados por quienes gozan de posiciones de poder dentro de la familia, el poder en la mayoría de los casos dado por la fuerza física o la posesión económica. "[...] La violencia ya sea conyugal, progenito filial, fraternal y parental extensa, responde a lógicas particulares y contiene sentidos, significados inherentes a la estructuración de cada grupo familiar" (Cuartas, 2012, p. 92).

Las estructuras familiares, actualmente entendidas de acuerdo con las nuevas formas de tipologías de la familia ${ }^{1}$, dejan ver cómo, en muchas estructuras familiares, los hombres de las familias son quienes aún asumen el rol de verdugos, pues socialmente conservan los privilegios de tener salarios más altos y mayores posibilidades de empleo (Badel \& Peña, 2010), sumado a que la sociedad todavía normaliza la relación hombre/violencia como algo rutinario.

A este propósito, el consumo de drogas y alcohol, la normalización de la violencia e inequidad de género y la inseguridad económica (Eisner, 2002), aparecen como factores de riesgo $\mathrm{y}$ determinantes de violencia intrafamiliar.

Lainseguridad económica es una constante en el caso de mujeres víctimas de la violencia doméstica (García-Moreno, Jansen, Ellsberg, Heise, \& Watts, 2005), ya que muchas carecen de autonomía económica y esto las condiciona a soportar el maltrato (García-Moreno, Jansen, Ellsberg, Heise, \& Watts, 2006). Este tema es tratado también por Ripoll, García y Ramírez (2014) quienes señalan que la distribución desigual de bienes en la sociedad o inseguridad económica, es una de las principales causas perpetuadores de la violencia, plantean que la posesión de vivienda es un medio de protección ante la violencia intrafamiliar. El acceso a bienes económicos como factor en las cifras de casos de violencia intrafamiliar explica no solo por qué son las mujeres las principales víctimas, sino también por qué lo son los niños, adultos mayores y discapacitados (Kitzmann, Gaylord, Holt, \& Kenny, 2003), por lo cual se deberían orientar políticas públicas hacia la prevención de esta desigualdad social generadora de actos violentos.

En el caso rural, se presentan menos casos de maltrato a mujeres (Gómez-López, Murad, \& Calderón, 2013). Esto se puede explicar por una dificultad hacia la denuncia (Escobedo, Bejarano, Castilla, \& León, 1997). La normalización de la violencia como forma legítima de comunicación en el caso de los hombres está relacionada con una formación social vigente en el país, que vincula a los hombres con la utilización de la violencia (Echandía, 1999; Pecaut \& González, 1997). 


\section{Clasificación de violencia de género.}

Aunque existe evidencia de la violencia masculina intrafamiliar, las cifras que reportan las instituciones son producto de las denuncias; en el caso de violencia intrafamiliar hacia los hombres, son pocos los que denuncian. Este silencio sugiere un campo de estudio considerable, ya que los hombres también son víctimas de su propia dominación (Bourdieu \& Jorda, 2000). La literatura refuerza de cierta manera este silencia pues formula un enfoque de género que relaciona violencia de género con mujer.

En la segunda mitad del siglo $\mathrm{XX}$, surgieron en el país reformas jurídicas que permitieron que las condiciones entre hombres y mujeres fueran más equitativas: en 1957, el derecho de las mujeres a ser ciudadanas, a elegir y ser elegidas; la Ley 75 de 1968 sobre la paternidad responsable, la Ley 20 de 1974 que dándole potestad a los dos cónyuges por igual, exigió la fidelidad como compromiso de los dos y no únicamente de la mujer (Cisneros-Trujillo, 2006); estos progresos en el ámbito jurídico han permitido construir el marco de pensamiento con el cual se entiende a la violencia como un asunto de interés público y no solo de interés privado.

La violencia intrafamiliar se convierte en un asunto público por las repercusiones sociales del fenómeno, y es allí cuando las instituciones logran intervenir en los conflictos a través de la denuncia y penalización de los mismos (Rico, 1996), no obstante y a pesar de los avances jurídicos, las tensiones entre lo público y lo privado hacen más difícil la sanción (Ortega, 2005). Es el caso de muchas mujeres que, al denunciar a su agresor, se encuentran con autoridades que justifican el hecho o le invitan a dialogar de manera privada el asunto "para evitar los trámites judiciales", ya difíciles de atender. A esto se suma que "los hechos de violencia en las historias masculinas y masculinizantes de los hombres aparecen minimizados y justificados de diversas formas, como manera de imponer su propia jerarquía de significaciones que, en ocasiones, logra calar en los resultados y la dirección de la balanza de la justicia” (Pineda \& Otero, 2004, p. 24).

Lo anterior se puede ver reflejado en los estereotipos de género que refuerzan la idea de que existe el derecho por parte del compañero sentimental o el esposo, de controlar los comportamientos de su pareja por medio de actos violentos (Sierra et al., 2006). De esta circunstancia nace el hecho de aceptar los estereotipos de género que son cómplices y que perpetúan relaciones inequitativas, siendo destructores de identidad de género para las nuevas generaciones, que validan su identidad en virtud de la inequidad y la violencia.

\section{Violencia, inseguridad y victimiza- ción de género.}

De acuerdo con las cifras de los entes de control, es indiscutible que las mujeres son las más afectadas por la violencia doméstica, como si un factor de riesgo para sufrir agresiones fuera el solo hecho de ser mujer; sin embargo, es necesario entender por qué son las mujeres, llevando el tema hacia una feminización de la violencia, y para esto valdría la pena hacer más estudios desde los hombres.

Por ejemplo, en el estudio "Necesidades, expectativas y sueños sobre la relación de pareja en hombres remitidos para atencion psicológica por denuncias de violencia intrafamiliar" (Medina, Arévalo, \& Durán, 2015) se observa cómo muchos de los hombres victimarios fueron víctimas de diferentes tipos de violencia en su infancia, lo que les dio a entender la violencia como un sistema normalizado de comunicación, corrección y expresión masculina, por lo cuál valdría la pena estudiar los casos de maltrato en niños y niñas.

La asociación del estereotipo de lo masculino (macho, viril) con el uso de la violencia, 
conduce a una minimización de los hechos cuando son las mujeres quienes los denuncian. A este propósito se suma que los victimarios transfieren la responsabilidad de sus actos (culpa) hacia las víctimas y dicha estrategia, lamentablemente, cuenta con la complicidad de algunos funcionarios de los organismos de control. Las actuaciones jurisdiccionales otorgadas a las comisarías de familia a través de la Ley 575 de 2000, con el objetivo de descongestionar los juzgados de familia y promiscuos municipales, trasladó sin duda una gran demanda de usuarios a estas entidades, sobre la base de considerar los delitos de violencia intrafamiliar, como delitos menores (Pineda \& Otero, 2004).

Bien pareciera por todo lo anterior, que se deben crear redes fuertes de apoyo,en las instituciones para que los funcionarios no minimicen los delitos y para que inviten a las mujeres maltratadas a manifestarse de manera pública, evitando que quede en el ámbito privado donde la violencia sin sanción se sigue propagando. Para generar cambios en la relación víctima/victimario (donde en casi la mayoría de casos las víctimas son mujeres), es necesario concentrarse en los hombres victimarios desde las políticas públicas, ya que las respuestas de atención institucional conservan un enfoque ginocéntrico (Velásquez y Ospina, 2011).

La tendencia que caracteriza la violencia y victimización en Colombia, revela comportamientos asociados a las condiciones municipales de seguridad y conflicto regional (Sánchez et al., 2003), esto obedece a las características económicas y a la presencia de acciones de conflicto municipal que diferencian regiones entre sí. Sin embargo dichas condiciones han cambiado en los últimos años, producto de la menor intensidad del conflicto armado, la industrialización regional y la dependencia en la extracción mineral en las regiones, mostrando evolución en las formas de violencia y victimización regional que afectan de forma diferenciada a hombres, mujeres, niños y adultos mayores (Carranza, Dueñas, \& González, 2011).
Por otra parte, en cuanto a la población infantil (Hartjen \& Priyadarsini, 2003) y controlando variables de edad, género, grados de estudio, ingreso familiar, involucramiento con la familia, escuela y compañeros, se explica el comportamiento asocial y tendencia criminal de niños y niñas; sumado a esto, se encuentra que existe una relación entre entorno y acciones violentas, delincuencia en general y menor escalas de delitos, ejercidas tanto por niños como por niñas. Mientras que las variables económicas no se relacionan con escalas delictivas, en general, vivir en un lugar ajeno a su hogar, es una causa de delincuencia principalmente en niños.

Otras variables a observar en la ocurrencia de acciones violentas o criminales, en sitios públicos o privados, son género, raza, edad, estatus y gravedad del delito (Miller, 2007). Los resultados arrojan los estudios, es que los delincuentes hombres tienen mayor probabilidad de estar involucrados en delitos en lugares públicos más que en privados; por el contrario, en las mujeres aumenta la probabilidad de cometer delitos en sitios privados, como residencias. Hay otro aspecto que surge y es que mientras los hombres tienden a delinquir en grupo, las mujeres prefieren cometer crímenes individualmente.

\section{Aproximación teórica}

Para una persona que se encuentra en una relación de convivencia y que debe decidir si se mantiene en ella, su nivel de utilidad se maximiza sujeto a las restricciones de la otra persona y permite un nivel de equilibrio donde la utilidad es creciente a partir del consumo de las variables que definen el nivel de utilidad. En el caso de una relación con abuso físico, el uso de formas de violencia incrementa la utilidad de agresor $U^{M}$ hacia la mujer $U^{M}$ tal y como lo presenta (Farmer \& Tiefenthaler, 1997).

$$
\operatorname{Max}_{V, C^{M}} U^{M}\left(S(V), C^{M}, \eta\right) \text { s. } a: \bar{U}^{W}=U^{W}
$$

(Ecuación 1) 
Donde: $\mathrm{S}(\mathrm{V})$ incorpora factores de autoestima que incrementan la utilidad cuando se presenta agresión física hacia otra persona; $C^{M}$ : consumo; ๆ: capital marital ${ }^{2} ; U^{W}=U^{W}\left(V, C^{W}, \eta, U^{M}\right), ; \bar{U}^{W}$ : utilidad exógena o punto de amenaza. Las condiciones de primer orden resultantes

$$
\begin{aligned}
& \frac{U_{V}^{M}}{U_{C^{M}}^{M}}=-\frac{U_{V}^{W}}{U_{C^{W}}^{W}} \\
& U^{W}=\bar{U}^{W}\left(C^{W}\right)
\end{aligned}
$$

\section{Gráfica 1: Elección óptima por transferencia} de violencia y consumo.
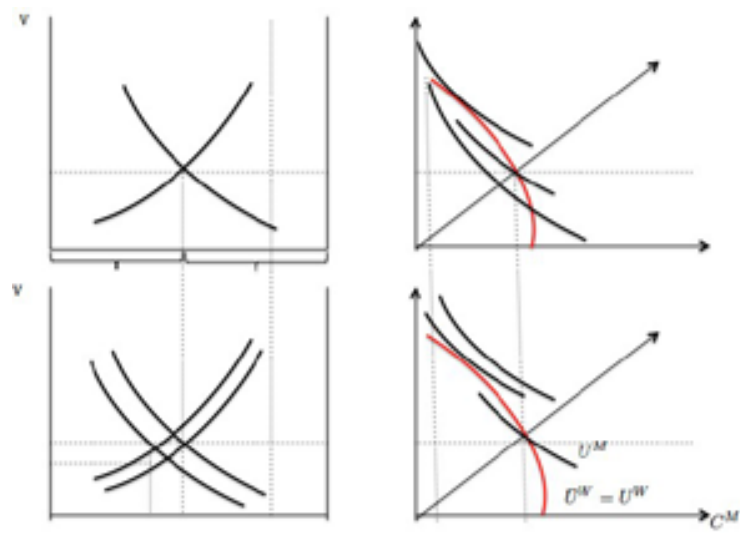

Fuente: Elaboración propia.

La ilustración anterior, muestra la solución interior asociada a la decisión óptima de la persona que debe disponer si permanece en una relación de convivencia cuando enfrenta violencia en el hogar: la expresión de la izquierda corresponde a la ecuación 2, la frontera de posibilidades representada en la curva de la gráfica y el término de la derecha representa la pendiente de la curva de indiferencia. Los términos de la ecuación 3 indican que el nivel de utilidad de la persona agredida supone el consumo de las variables al interior del hogar y que el agresor mantiene constante su nivel de utilidad. Dicha solución interior representa la tasa marginal de sustitución del agresor y la víctima, dada por el nivel de violencia y consumo.

Siendo la expresión de la izquierda la frontera de posibilidades y el término de la de- recha la representación de la pendiente de la curva de indiferencia, donde los términos de la función indican que el castigo marginal debe ser mayor a la diferencia de la utilidad marginal obtenida por la víctima, de lo contrario cometer agresiones será una opción para los agresores. Esto sugiere que cometer agresiones depende del retorno relativo entre violencia y consumo individual: a menor nivel de ingreso o de consumo con respecto a la pareja, aumentan las intenciones de perpetrar agresiones.

En el caso de la mujer afectada por violencia masculina, su decisión impacta la solución de equilibrio parcial y es producto del proceso de optimización inicial mediante un cambio en la solución no cooperativa, incluyendo trabajo y menor tiempo libre, que termina endogenizandose en su nivel de ingreso: unos mejores recursos económicos generan una reducción de los actos violentos de los que sonvíctimas. La magnitud en el cambio de las variables involucradas en el nivel de utilidad puede tener mayor impacto, dependiendo del nivel de violencia que lleva a la mujer a tomar una decisión, lo que le permite tener una curva de indiferencia más alta, muy distinta a la que puede tener al interior del hogar.

\section{Estimación del modelo.}

Retomando, ciertas variables actúan como incentivos para que las mujeres que padecen violencia intrafamiliar, salgan de esas relaciones; a través de la educación y del trabajo mejoran sus ingresos, incluso por encima del agresor. Para los casos en los que se mantienen relaciones estables, las variables que motivan a ello son un mayor nivel de escolaridad y porque existen ingresos adicionales en el hogar por actividades paralelas a un trabajo regular.

Dicho esto, trabajo, educación, ingresos del hogar, estado civil, presencia de hijos en el hogar, edad y tiempo de la relación, son variables que inciden en el aumento del nivel de utilidad para reducir el número de agresiones del jefe del hogar hacia la mujer. 


\section{Datos y variables.}

Los datos entre 2012-2015, corresponden a la Encuesta de Convivencia y Seguridad Ciudadana -ECSC- del Departamento Administrativo Nacional de Estadística (DANE) realizada, cada año, a personas diferentes de una muestra de municipios representativos. Se construyeron las siguientes variables: agresiones físicas del jefe del hogar hacia la mujer, ingreso de hombre y mujer, si el encuestado es casado(a), si existen hijos y menores en el hogar, edad de la mujer, años de educación de la mujer, tiempo de relación con el jefe del hogar y por último, otros ingresos del hogar. Por las características de la encuesta (representa hogares que no son comparables en el tiempo y los cuestionarios de las preguntas cambian de un año a otro), se dificulta realizar estimaciones conjuntas.

\section{Modelo empírico.}

Se sugiere una correspondencia entre agresiones físicas y variables como ingreso, ocio, educación y tipo de relación con el jefe del hogar. Para evaluar dicha relación, la mujer víctima de agresiones presenta una función no observable de utilidad indirecta que se plantea de la siguiente manera:

$v_{w \mid v}=x_{i} \beta+w_{(w \mid v), t} \gamma+n_{(w \mid v)} \eta$

(Ecuación 4)

Al no observar la utilidad indirecta se considera la siguiente especificación con los datos de encuestados por municipios que permiten caracterizar a partir de una estimación Tobit las decisiones de las mujeres víctimas de agresiones físicas por el jefe del hogar.

$V W_{W \mid V, i, t}^{*}=x_{i, t} \vartheta+\lambda_{i, t}+\epsilon_{i, t}$

(Ecuación 5)

$$
\begin{aligned}
& V W_{W \mid V, i, t}^{*}=\operatorname{Tr}_{i, t}^{M}+\operatorname{Tra}_{i, t}^{W}+E s t_{i, t}+H i j_{i, t} \\
& +E d_{i, t}^{W}+E s c^{W}+\operatorname{Tim}_{i, t}^{W}+E s t_{i, t}
\end{aligned}
$$

(Ecuación 6)
Donde: $V W_{W \mid v, i}^{*}$, es el número de veces que la mujer afirma estar involucrada en riñas o peleas que implican violencia física en los últimos meses con el jefe del hogar, $\operatorname{Tr}_{i, t}^{M}$ indica si el hombre trabaja o trabaja y estudia, $\operatorname{Tr}_{i, t}^{M}$ indica si la mujer trabaja o trabaja y estudia, $E t_{i, t}$ indica si la persona es casado(a), $H i j_{i, t}$ indica si en el hogar existen hijos y menores de edad, $E d_{i, t}^{W}$ edad en años de la mujer, $E s c^{W}$ años de educación de la mujer, $\operatorname{Tim}_{i, t}^{W}$ tiempo en meses de relación, $E s t_{i, t}$ otros ingresos del hogar representado en el estrato.

\section{Resultados.}

La Tabla 1 (Anexo 1) muestra las estadísticas descriptivas de los datos utilizados, mientras que la Tabla 2 (Anexo 2) son resultados de las estimaciones, por año, usando todos los datos disponibles y controlando únicamente por los reportes de mujeres involucradas en riñas o peleas que implican violencia física por el cónyuge jefe del hogar.

En todas las estimaciones cuando se usa la muestra completa, las variables de ocupación de hombres y mujeres, el nivel de ingreso del hogar definido por estrato social, la presencia de hijos, el tiempo de duración de la relación actual, los años de escolaridad y la edad de la mujer, inciden en la probabilidad de reducir los actos violentos hacia la mujer por parte del jefe del hogar.

Mientras que al controlar solo por los casos de mujeres, víctimas recientes de violencia directa inflingida por el jefe del hogar, estas pocas variables: estar casadas, la presencia de hijos y menores en el hogar y que las mujeres se encuentren trabajando, influyen reduciendo la probabilidad de que sean víctimas de agresiones.

Los victimarios, sin importar las diferentes estructuras familiares, son en su mayoría los jefes del hogar; esto podría ser explicado no solo por el estatus otorgado dentro de la familia sino también por sus posibilidades económicas. Las 
víctimas, por su parte, sufren cierto grado de inseguridad económica o de dependencia afectiva. Los victimarios presentan niveles más bajos de escolaridad, pese a que el nivel de escolarización no es necesariamente un determinante de violencia en los sujetos: las cifras muestran que existen niveles de escolarización similares en las diferentes zonas donde la mayoría de la población ha alcanzado a tener educación media, sin embargo este si es un agravante.

Las familias que sufren violencia por parte del jefe del hogar, pueden no tener una estructura familiar fija, están compuestas por parejas que viven bajo el mismo techo y no necesariamente están casadas, esto muestra que la diversificación de la familia es difícil de afrontar para quienes tienen convivencia, porque la violencia hacia la mujer está subordinada a las condiciones de la casa, pero la decision de mantenerse en un hogar con violencia dependerá de la valoración del consumo de las variables relevantes para la víctima del maltrato.

\section{Conclusiones}

- Los resultados apoyan la relación entre agresiones físicas hacia la mujer por parte del jefe del hogar y los niveles de ocupación en hombres y mujeres, nivel de escolaridad y edad de la mujer, ingreso del hogar, tiempo de permanencia de la relación de pareja, estado civil de los integrantes de la misma y presencia de hijos en el hogar.

- Las variables asociadas con ingreso, escolaridad, ocupación y educación de la mujer, son importantes para explicar la disminución de la probabilidad de ocurrencia de violencia física del hombre hacia esta.

- Una mujer puede verse involucrada en riñas y peleas con el jefe del hogar, y estas pueden aumentar e involucrar agresiones físicas a pesar de que ella cuente con un trabajo y tenga hijos con este. Este punto explica cómo la violencia de género puede tener raíces en la condición laboral, la ocupación y la brecha salarial con el jefe del hogar.

- Los resultados invitan a estudiar el impacto que tienen las condiciones económicas, sociales y el tiempo de convivencia que lleva la pareja, sobre las diferentes formas de violencia ejercidas contra la mujer, dentro y fuera del hogar.

- Queremos destacar lo importante que es establecer vínculos de largo plazo antes de pasar a convivir, porque a medida que se quiebra la unión altruista que persiguen el matrimonio y la convivencia con la pareja, también se daña la condición de equilibrio cooperativo, lo que incita a que se presenten hechos violentos que antes de la convivencia no se consideraban posibles.

En términos generales, la estática comparativa del modelo planteado y la aproximación empírica se ajustan a los resultados encontrados previamente: factores como que la mujer genere un ingreso superior al de su pareja, que se perciban otros ingresos en el hogar distintos a los obtenidos salarialmente, la estabilidad de la relación, que la pareja se encuentre unida con vínculo matrimonial y que las obligaciones económicas generadas por los hijos puedan ser cubiertas con los ingresos disponibles, son en conjunto buenos estimadores de la disminución en la cantidad de agresiones físicas hacia la mujer por parte de sus parejas y podrían ser factores por los que hay menos cantidad de divorcios en regiones con presencia de familias económicamente estables.

\section{Notas}

1 Medicina Legal reconoce estructuras como: la familia nuclear, extensa, monoparental, superpuesta o recompuesta, pareja sin hijos, unipersonal y nuclear con amantazgo (Sierra, Macana, \& Cortes, 2006)

2 El capital marital corresponde a bienes físicos y materiales del hogar. Farmer \& Tiefenthaler (1997). 


\section{Referencias}

Acero, Á. R., Escobar, F., \& Castellanos, G. (2007). Factores de riesgo para violencia y homicidio juvenil. Revista Colombiana de psiquiatría, 36(1), 78-97.

Anderson, E. A., \& Diaz, J. (1996). Using process control chart techniques to analyse crime rates in Houston, Texas. Journal of the Operational research Society, 47(7), 871-881.

Badel, A., \& Peña, X. (2010). Decomposing the gender wage gap with sample selection adjustment: Evidence from Colombia. Revista de Análisis Económico, 25(2), 169-191. Recuerado de http://www. scielo.cl/pdf/rae/v25n2/art07.pdf

Boucekkine, R., Croix, D., \& Licandro, O. (2002). Vintage human capital, demographic trends, and endogenous growth. Journal of Economic Theory, 104(2), 340-375.

Bourdieu, P., \& Jorda, J. (2000). La dominación masculina (Vol. 3). Barcelona: Anagrama.

Caicedo, C. (2005). Lucha contra la violencia intrafamiliar: perspectivas desde la experiencia colombiana. París: Asociación mundial Escuela Instrumento de Paz.

Carranza, J. E., Dueñas, X., \& González, C. (2011). Análisis empírico de la relación entre la actividad económica y la violencia homicida en colombia. Estudios Gerenciales, 27(119), 59-78.

Chen, X., Thrane, L., Whitbeck, L. B., Johnson, K. D., \& Hoyt, D. R. (2007). Onset of conduct disorder, use of delinquent subsistence strategies, and street victimization among homeless and runaway adolescents in the midwest. Journal of interpersonal violence, 22(9), 1156-1183.
Cisneros-Trujillo, C. (2006). La violencia intrafamiliar política criminal de Estado. Universitas Estudiantes, 3, 203-223.

Cuartas, M. Y. G. (2012). La violencia intrafamiliar una problemática que requiere pensarse desde lo interinstitucional. Eleuthe$r a, 7,90-103$.

Departamento Administrativo Nacional de Estadística (DANE). (2015) Encuesta de Convivencia y Seguridad Ciudadana. Recuperado de https://www.dane.gov.co/ index.php/estadisticas-por-tema/seguridad-y-defensa/encuesta-de-convivenciay-seguridad-ciudadana-ecsc/ecsc-2015

Echandía, C. (1999). Expansión territorial de las guerrillas colombianas: geografía, economía y violencia. En M. Deas \& M. Llorente (Comps.), Reconocer la guerra para construir la paz (pp.99-149). Bogotá: Cerec; Norma; Ediciones Uniandes.

Eisner, M. (2002). Crime, problem drinking, and drug use: Patterns of problem behavior in cross national perspective. The Annals of the American Academy of Political and Social Science, 580(1), 201-225.

Escobedo, L., Bejarano, J. A., Castilla, C. E., \& León, E. (1997). Colombia: inseguridad, violencia $y$ desempeño económico en las áreas rurales. Fondo Financiero de Proyectos de Desarrollo. Bogotá: Universidad Externado de Colombia; Fondo Financiero de Proyectos de Desarrollo.

Farmer, A., \& Tiefenthaler, J.(1997). An economic analysis of domestic violence. Review of Social Economy, 55(3), 337-358.

García-Moreno, C., Jansen, H., Ellsberg, M., Heise, L., \& Watts, C. (2005). Who multi country study on women's health and domestic violence against women: initial results on prevalence, health outcomes 
and women's responses. Suiza: World Health Organization.

García-Moreno, C., Jansen, H., Ellsberg, M., Heise, L., \& Watts, C. (2006). Prevalence of intimate partner violence: findings from the who multi country study on women's health and domestic violence. The Lancet, 368(9543), 1260-1269. doi: 10.1016/ S0140-6736(06)69523-8

Gómez-López, C., Murad, R., \& Calderón, M. (2013). Historias de violencia, roles, prácticas y discursos legitimadores. Violencia contra las mujeres en Colombia, 2000-2010. Recuperado de https://www. minsalud.gov.co/sites/rid/Lists/BibliotecaDigital/RIDE/INEC/INV/7\%20-\%20VIOLENCIA $\% 20$ CONTRA $\% 20 L A S \% 20$ MUJERES\%20EN\%20COLOMBIA.pdf

Gottfredson, M. R., \& Hirschi, T. (1990). A general theory of crime. Stanford: Stanford University Press.

Hartjen, C. A., \& Priyadarsini, S. (2003). Gender, peers, and delinquency a study of boys and girls in rural France. Youth \& Society, 34(4), 387-414.

Kitzmann, K. M., Gaylord, N. K., Holt, A. R., \& Kenny, E. D. (2003). Child witnesses to domestic violence: a meta analytic review. Journal of consulting and clinical psychology, 71(2), 339-352.

Lafaurie, M. M. (2015). La violencia intrafamiliar contra las mujeres en Bogotá: una mirada de género. Revista Colombiana de Enfermería, 8(8), 98-111.

Ley 75 de 1968. Por la cual se dictan normas sobre filiación y se crea el Instituto Colombiano de Bienestar Familiar. Diario oficial No. 32.682. Congreso de la República de Colombia, diciembre de 1968.
Ley 20 de 1974. Por el cual se prueba el Concordato y el Protocolo Final entre la República de Colombia y la Santa Sede suscrito en Bogotá el 12 de julio de 1973. Diario oficial No. 34.234. Congreso de la República de Colombia, diciembre de 1974.

Ley 294 de 1996. Por la cual se desarrolla el artículo 42 de la Constitución Política y se dictan normas para prevenir, remediar y sancionar la violencia intrafamiliar. Diario oficial No. 42.836. Congreso de la República de Colombia, julio de 1996.

Ley 575 del 2000. Por medio de la cual se reforma parcialmente la Ley 294 de 1996. Diario oficial No. 43.889. Congreso de la República de Colombia, febrero de 2000.

Medina, A., Arévalo, A., \& Durán, A. (2015). Necesidades, expectativas y sueños sobre la relación de pareja en hombres remitidos para atencion psicológica por denuncias de violencia intrafamiliar. Universitas Psychologica, 14(1), 15-28. doi: 10.11144/Javeriana.upsy14-1.nesr

Miller, K. (2007). Traversing the spatial divide? gender, place, and delinquency. Feminist Criminology, 2(3), 202-222.

Molina, D., \& Ospina, A. (2011). La posición masculina ante la violencia intrafamiliar: una pregunta sobre la afectividad silenciada. Revista Virtual Universidad Católica del Norte, 32, 181-200.

Ortega, L. R. (2005). Buenas prácticas para la erradicación de la violencia doméstica en la región de América Latina y el Caribe (Comisión Económica para América Latina y el Caribe -[CEPAL], Serie Mujer y Desarrollo No. 75). Santiago de Chile: Naciones Unidas.

Pallitto, C. C., \& O'Campo, P. (2005). Community level effects of gender inequality on 
intimate partner violence and unintended pregnancy in Colombia: testing the feminist perspective. Social science \& medicine, 60(10), 2205-2216.

Pecaut, D., \& González, L. (1997). Presente, pasado y futuro de la violencia en Colombia. Desarrollo Económico, 36(144), 891-930.

Pineda, J., \& Otero, L. (2004). Género, violencia intrafamiliar e intervención pública en Colombia. Revista de estudios sociales, 17, 19-31.

Prendergast, M., Huang, D., \& Hser, Y. I. (2008). Patterns of crime and drug use trajectories in relation to treatment initiation and 5 -year outcomes an application of growth mixture modeling across three data sets. Evaluation review , 32(1), 59-82. doi: 10.1177/0193841X07308082

Quiñones, M. C., Arias, Y., Delgado, E. M., \& Tejera, A. J. (2011). Violencia intrafamiliar desde un enfoque de género. Mediciego, 17(2). Recuperado de http:// www.medigraphic.com/pdfs/mediciego/ mdc-2011/mdc112za.pdf

Rico, M. N. (1996). Violencia de género: un problema de derechos humanos (Comisión Económica para América Latina y el Caribe -[CEPAL], Serie Mujer y Desarrollo No. 16). Recuperado de http://www.cepal.org/mujer/noticias/ paginas/3/27403/violenciadegenero.pdf
Ripoll, J., García, S., \& Ramirez, H. (2014). Vivienda/violencia: intersecciones de la vivienda y la violencia intrafamiliar en Ciudad Bolívar, Bogotá. Revista de Estudios Sociales, 50, 71-86.

Sánchez, F., Díaz, A. M., \& Formisano, M. (2003). Conflicto, violencia y actividad criminal en colombia: un análisis espacial (Centro de Estudios sobre Desarrollo Económico -CEDE, Documento No. 2003-05). Recuperado de https:// core.ac.uk/download/pdf/6517007.pdf

Savoie, J., Bédard, F., \& Collins, K. (2006). Neighbourhood characteristics and the distribution of crime on the island of Montréal (Canadian Centre for Justice Statistics, Crime and Justice Research Paper Series, Catalogue no. 85-561-M No. 011). Ottawa: Statistics Canada. Recuperado de http://www.statcan.gc.ca/pub/85561-m/85-561-m2008011-eng.pdf

Sierra, R., Macana, N., \& Cortes, C. (2006). Impacto social de la violencia intrafamiliar. Revista Forensis, 81-150. Recuperado de http://www.medicinalegal.gov.co/ documents/10180/33850/9+Violenciaintr afamiliar.pdf/10708fa9-efb1-4904-a9e6$36377 \mathrm{ca} 8 \mathrm{a} 912$

Watts, R. E. (1931). The influence of population density on crime. Journal of the American Statistical Association, 26(173), 1120. 
Anexo 1.

Tabla 1: Análisis descriptivo

\begin{tabular}{|c|c|c|c|c|c|c|c|c|}
\hline \multirow[b]{2}{*}{ Variable } & \multicolumn{2}{|c|}{ [2012] } & \multicolumn{2}{|c|}{ [2012] } & \multicolumn{2}{|c|}{ [2013] } & \multicolumn{2}{|c|}{ [2013] } \\
\hline & Mean & Std. Dev. & Mean & Std. Dev. & Mean & Std. Dev. & Mean & Std. Dev. \\
\hline numviofem & 0.0030214 & 0.0768917 & $1,565,868$ & .7866203 & 0.0042562 & 0.1275696 & $1,789,731$ & $1,912,239$ \\
\hline homtra & 0.2474928 & 0.4315567 & 0 & 0 & 0.2405254 & 0.4274038 & 0 & 0 \\
\hline mujtra & 0.1676545 & 0.3735603 & 0.3742515 & 0.4846551 & 0.1723803 & 0.3777118 & 0.4327628 & 0.4960653 \\
\hline married & 0.1856268 & 0.3888063 & 0.4221557 & 0.4946441 & 0.1868582 & 0.3897988 & 0.4376528 & 0.4967052 \\
\hline prehij & 0.0779087 & 0.2680287 & 0 & 0 & 0.0767853 & 0.2662513 & 0 & 0 \\
\hline edmuj & 17.65759 & 22.51598 & 39.11677 & 12.43801 & 17.7212 & 22.61445 & 38.56479 & 12.74222 \\
\hline educmuj & 4.70457 & 5.680776 & 9.931138 & 3.638886 & 4.75455 & 5.72767 & 10.02445 & 3.778732 \\
\hline tirel & 84.60194 & 42.17566 & 75.01796 & 43.49526 & 83.72049 & 42.5469 & 74.49389 & 44.33562 \\
\hline estra & 2.208169 & 1.092636 & 2.188623 & 1.047114 & 2.231321 & 1.116988 & 2.03423 & 1.061262 \\
\hline $\mathbf{N}$ & \multicolumn{2}{|c|}{173100} & \multicolumn{2}{|c|}{334} & \multicolumn{2}{|c|}{171986} & \multicolumn{2}{|c|}{409} \\
\hline
\end{tabular}

\begin{tabular}{|c|c|c|c|c|c|c|c|c|}
\hline \multirow[b]{2}{*}{ Variable } & \multicolumn{2}{|c|}{$[2014]$} & \multicolumn{2}{|c|}{ [2014] } & \multicolumn{2}{|c|}{ [2015] } & \multicolumn{2}{|c|}{ [2015] } \\
\hline & Mean & Std. Dev. & Mean & Std. Dev. & Mean & Std. Dev. & Mean & Std. Dev. \\
\hline numviofem & 0.004564 & 0.1242792 & $1,831,081$ & 169,072 & .002179 & .0804702 & $1,550,607$ & $1,488,598$ \\
\hline homtra & 0.3132733 & 0.4638258 & 0 & 0 & .3126298 & .4635662 & 0 & 0 \\
\hline mujtra & 0.2313004 & 0.4216652 & 0.4076577 & 0.4919532 & .2348962 & .4239351 & 0.4898785 & 0.5009126 \\
\hline married & 0.2348202 & 0.4238876 & 0.3603604 & 0.4806465 & .2352774 & .4241733 & 0.2874494 & 0.4534918 \\
\hline prehij & 0.0236566 & 0.1519773 & 0 & 0 & .0243561 & .1541526 & 0 & 0 \\
\hline edmuj & 22.15753 & 24.09322 & 37.4482 & 13.40713 & 22.33495 & 24.29985 & 35.84211 & 11.5461 \\
\hline educmuj & 7.059299 & 6.832648 & 13.03604 & 2.06411 & 7.085477 & 6.848552 & 13.28745 & 2.201802 \\
\hline tirel & 85.79395 & 42.49055 & 74.94595 & 44.05298 & 86.61988 & 42.40183 & 75.35223 & 43.56139 \\
\hline estra & 2.221875 & 1.108726 & 2.045045 & 0.9299374 & 2.197876 & 1.091091 & 1.919028 & 0.9550477 \\
\hline $\mathbf{N}$ & \multicolumn{2}{|c|}{178132} & \multicolumn{2}{|c|}{444} & \multicolumn{2}{|c|}{175767} & \multicolumn{2}{|c|}{247} \\
\hline
\end{tabular}


Tabla 2: Resultados de estimaciones 2012-2015

\begin{tabular}{|c|c|c|c|c|c|c|c|c|}
\hline $\begin{array}{c}\text { Variables } \\
\text { Explicativas }\end{array}$ & [2012] & [2012] & [2013] & [2013] & [2014] & [2014] & [2015] & [2015] \\
\hline \multirow[t]{3}{*}{ homtra } & $-10.133 * *$ & 0.0001 & $-27.5909^{* *}$ & 0.0001 & $-30.6791 * * *$ & 0.0001 & $-31.3967 * * *$ & 0.0001 \\
\hline & $(-0.1065)$ & $(0.002)$ & $(0.7257)$ & $(0.002)$ & $(0.002)$ & $(0.002)$ & $(0.002)$ & $(0.002)$ \\
\hline & {$[-5.06]$} & {$[1.23]$} & {$[-6.05]$} & {$[0.23]$} & {$[0.23]$} & {$[1.23]$} & {$[0.13]$} & {$[0.23]$} \\
\hline \multirow[t]{3}{*}{ mujtra } & 0.1971 & 0.1778 & $1.2843^{* * *}$ & $1.0253 * * *$ & -0.2124 & $-0.3896 * * *$ & $1.5641^{* * *}$ & 1.1037 \\
\hline & $(0.1484)$ & $(0.2301)$ & $(0.4726)$ & $(0.5380)$ & $(0.3875)$ & $(0.4407)$ & $(0.7506)$ & $(0.7431)$ \\
\hline & [1.33] & {$[0.77]$} & {$[2.72]$} & [1.91] & {$[-0.55]$} & {$[-0.88]$} & [2.08] & [1.49] \\
\hline \multirow[t]{3}{*}{ married } & $1.067 * * *$ & -0.0814 & $3.7385 * * *$ & $1.4606^{* * *}$ & $1.7678^{* * *}$ & 0.1381 & $1.4856^{* * *}$ & -0.1430 \\
\hline & $(0.1665)$ & $(0.2521)$ & $(0.7296)$ & $(0.6693)$ & $(0.4276)$ & $(0.48272)$ & $(0.7176)$ & $(0.8067)$ \\
\hline & [6.41] & {$[-0.32]$} & [5.12] & [2.18] & [4.13] & {$[0.29]$} & [2.07] & {$[-0.18]$} \\
\hline \multirow[t]{3}{*}{ prehij } & $-9.1672 * *$ & 0.0001 & $-25.2804 * * *$ & 0.0001 & $-29.3030 * * *$ & 0.0001 & $-30.3087^{* * *}$ & 0.0001 \\
\hline & $(0.0956)$ & $(0.001)$ & $(0.0042)$ & $(0.002)$ & $(0.001)$ & $(0.002)$ & $(0.001)$ & $(0.002)$ \\
\hline & {$[-4.16]$} & {$[0.23]$} & {$[-4.05]$} & {$[0.12]$} & {$[0.03]$} & {$[1.23]$} & {$[0.02]$} & {$[0.23]$} \\
\hline \multirow[t]{3}{*}{ edmuj } & -0.0006 & 0.0096 & $-0.0161 * * *$ & -0.0178 & $-0.0220 * * *$ & 0.0050 & $-0.0509 * * *$ & -0.0367 \\
\hline & $(0.0023)$ & $(0.0081)$ & $(0.0077)$ & $(0.0178)$ & $(0.0066)$ & $(0.0148)$ & $(0.0165)$ & $(0.0316)$ \\
\hline & {$[-0.30]$} & [1.19] & {$[-2.08]$} & {$[-1.00]$} & {$[-3.33]$} & {$[0.34]$} & {$[-3.08]$} & {$[-1.16]$} \\
\hline \multirow[t]{3}{*}{ educmuj } & $-0.0385 * * *$ & 0.0022 & $-0.1328 * * *$ & -0.0143 & $-0.2305^{* * *}$ & -0.0209 & $-0.2204 * * *$ & -0.0471 \\
\hline & $(0.0106)$ & $(0.0250)$ & $(0.0362)$ & $(0.0517)$ & $(0.0327)$ & $(0.0466)$ & $(0.0525)$ & $(0.0834)$ \\
\hline & {$[-3.62]$} & {$[0.09]$} & {$[-3.66]$} & {$[-0.28]$} & {$[-7.04]$} & {$[-0.45]$} & {$[-4.19]$} & {$[-0.56]$} \\
\hline \multirow[t]{3}{*}{ tirel2 } & $-0.0243 * * *$ & 0.0046 & $-0.0778 * * *$ & -0.0088 & $-0.0747 * * *$ & -0.0039 & $-0.0737 * * *$ & 0.0050 \\
\hline & $(0.0024)$ & $(0.0028)$ & $(0.0133)$ & $(0.0060)$ & $(0.0090)$ & $(0.0053)$ & $(0.0159)$ & $(0.0096)$ \\
\hline & {$[-9.91]$} & [1.64] & {$[-5.85]$} & {$[-1.45]$} & {$[-8.24]$} & {$[-0.74]$} & {$[-4.62]$} & {$[0.52]$} \\
\hline \multirow[t]{3}{*}{ estra } & $-2.0506 * * *$ & $-0.0867 * * *$ & $-5.9823 * * *$ & -0.2391 & $-5.0524 * * *$ & 0.0300 & $-9.1540 * * *$ & -0.5230 \\
\hline & $(0.2993)$ & $(0.1104)$ & $(1.1705)$ & $(0.2913)$ & $(0.7361)$ & $(0.2129)$ & $(2.2695)$ & $(0.3948)$ \\
\hline & {$[-6.85]$} & {$[-0.79]$} & {$[-5.11]$} & {$[-0.82]$} & {$[-6.86]$} & {$[0.14]$} & {$[-4.03]$} & {$[-1.32]$} \\
\hline $\mathrm{N}$ & 173100 & 334 & 171986 & 409 & 178132 & 444 & 175767 & 247 \\
\hline AIC & 3741.436 & 760.5676 & 4095.139 & 1070.836 & 4340.828 & 1180.869 & 1798.32 & 498.4251 \\
\hline $\mathrm{BIC}$ & 3811.867 & 787.2456 & 4165.525 & 1098.932 & 4411.46 & 1209.539 & 1868.859 & 522.9908 \\
\hline Log likelihood & -1863.718 & -373.28378 & -2040.5693 & -528.41814 & -2163.4139 & -583.43432 & -892.16011 & -242.21253 \\
\hline
\end{tabular}

